\title{
Amino Acid Metabolism during Starvation in Human Pregnancy
}

\author{
Phimip Felig, Young Jin Kim, Vincent Lynch, and Rosa Hendler \\ From the Department of Internal Medicine and the Department of Obstetrics \\ and Gynecology, Yale University School of Medicine, \\ New Haven, Connecticut 06510
}

A B S T R A T To evaluate the factors regulating gluconeogenesis in pregnancy, plasma amino acid levels were determined during the course of an 84-90 hr fast in physically healthy women studied during wk 16-22 of gestation (before undergoing therapeutic abortion), and in nonpregnant controls. The effect of pregnancy on the glycemic response to exogenous alanine administration during starvation was also investigated.

In the nonpregnant group fasting resulted in a 2- to 3fold increase in the levels of plasma valine, leucine, isoleucine, and $\alpha$-aminobutyrate, while the concentration of alanine and glycine fell. In the pregnant group, the levels of most amino acids were significantly reduced in the postabsorptive state. With starvation, the plasma concentration of alanine fell more rapidly in the pregnant group and was significantly below that of the nonpregnant subjects for the first $60 \mathrm{hr}$ of the fast. In contrast, a significant elevation in plasma glycine, serine, and threonine was observed in the pregnant group after $84 \mathrm{hr}$ of fasting, whereas similar increments were not demonstrable until after 10 days of fasting in previously studied nonpregnant obese subjects. Paralleling the changes in maternal plasma, amniotic fluid levels of valine, leucine, and isoleucine increased while that of alanine fell during the fast.

Although the plasma glucose concentration was lower in the pregnant group at termination of the fast, intravenous alanine administration $(0.15 \mathrm{~g} / \mathrm{kg})$, resulted in a prompt, comparable increase $(20-25 \mathrm{mg} / 100 \mathrm{ml})$ in plasma glucose in both groups of subjects.

It is concluded that $(a)$ pregnancy accelerates and exaggerates the hypoalaninemic and hyperglycinemic effects of starvation; (b) lack of key endogenous sub-

Dr. Felig is a Teaching and Research Scholar of the American College of Physicians.

Received for publication 10 September 1971 and in revised form 21 December 1971. strate rather than altered intrahepatic processes may limit hepatic gluconeogenesis in pregnancy and contribute to gestational hypoglycemia; $(c)$ maternal caloric deprivation profoundly alters the levels of amino acids in amniotic fluid.

\section{INTRODUCTION}

The influence of human pregnancy in exaggerating and accelerating the blood glucose, insulin, and ketone response to caloric deprivation has recently been demonstrated in women fasted during the second trimester (1). One of the major problems raised by these and similar studies in experimental animals $(2,3)$, concerns the mechanism whereby gluconeogenesis is regulated in gestation. Specifically, the basis for the failure of maternal gluconeogenic processes to keep pace with the total glucose requirements of the conceptus and mother has not been established. Thus during the course of a 3-4 day fast, maternal blood glucose levels fall markedly $(1,4)$, while urea nitrogen excretion, reflecting hepatic gluconeogenesis (5), fails to increase above the levels observed in the nongravid state (1). This gestational exaggeration of fasting hypoglycemia occurs in the face of maternal hypoinsulinemia and hyperketonemia, conditions which would be expected to augment hepatic gluconeogenesis $(6,7)$. The question thus may be raised as to whether pregnancy directly alters intrahepatic processes or alternatively affects the supply of glucose precursors so as to limit hepatic glucose production.

With respect to possible direct effects of gestation on the liver, studies with isotopically labeled glucose precursors in the starved pregnant rat have revealed an augmented rather than diminished capacity for conversion of exogenous substrate to glucose $(3,8)$. Similar data are not however available in man. As to the role of endogenous substrate presentation, the importance of 
the level of circulating alanine in the regulation of hepatic gluconeogenesis has been demonstrated in previous investigations in nonpregnant subjects fasted for prolonged periods (9). While data are available on the influence of pregnancy on plasma amino acid levels in the postabsorptive state (10-12) the effect of gestation on the amino acid response to starvation has not been examined. The significance of evaluating amino acid metabolism in the fasted pregnant state is underscored by the unique interaction which exists in this circumstance between maternal gluconeogenic mechanisms which require amino acids as glucose precursors, and placental transport processes which must meet fetal demands for amino acids for protein synthesis. Both processes thus are ultimately dependent on maternal protein stores and circulating amino acids as their source of substrate.

The present study was consequently designed to evaluate the factors regulating gluconeogenesis in human gestation by examining plasma amino acid levels in subjects fasted for $84-90 \mathrm{hr}$ in mid-pregnancy. In addition, as an indication of possible direct effects of gestation on maternal gluconeogenic potential during starvation, the blood glucose response to exogenous alanine administration has been determined. Finally, inasmuch as amniocentesis has become a useful diagnostic technique in the prenatal diagnosis of genetic disorders $(13,14)$, and since fasting has been demonstrated to alter amniotic fluid levels of glucose and ketones (15), the effects of caloric deprivation on the concentration of amino acids in amniotic fluid has been investigated.

\section{METHODS}

Subjects (Table I). Two groups of pregnant volunteers ("fasted" and "fed", Table I) were studied during the second trimester. They were in good physical health, and had been legally approved to undergo therapeutic abortion for psychiatric reasons by means of transabdominal intraamniotic installation of hypertonic saline. The nonpregnant group consisted of 11 healthy volunteers of similar age and weight (Table I). None of the pregnant or nonpregnant subjects had a history or evidence of obesity, diabetes,

TABLE I

Clinical Data on Subjects

\begin{tabular}{lccc}
\hline & \multicolumn{2}{c}{ Pregnant } & \\
\cline { 2 - 3 } & Fasted & Fed & Nonpregnant \\
\hline Number & 17 & 6 & 11 \\
Age, $y r$ & $15-26$ & $15-21$ & $19-23$ \\
Duration of pregnancy, $w k$ & $16-22$ & $16-22$ & - \\
Body weight, $k g$ & $47-67$ & $50-67$ & $53-70$ \\
\% Ideal weight* & $94-107$ & $96-107$ & $90-113$ \\
Weight loss, $\$ k g$ & $3.3 \pm 0.2$ & - & $3.1 \pm 0.4$ \\
\hline
\end{tabular}

* Based on Metropolitan Life Insurance tables, 1959.

$\ddagger$ During the course of 84-90 hr of fasting. thyroid or adrenal disorders, or liver disease. They were informed of the nature, purpose, and possible risks of the study before giving their voluntary consent. In the case of the pregnant group, the subjects were informed that therapeutic abortion would be performed regardless of their participation in the study.

Procedures. All of the subjects were hospitalized at the Clinical Research Center of the Yale-New Haven Hospital. 17 of the pregnant subjects and 7 of the nonpregnant subjects were studied during the course of an $84-90 \mathrm{hr}$ fast. Starvation was initiated after completion of the evening meal at 8 p.m. on the day of admission and was continued for 84-90 hr, until the morning of the 5th hospital day. During this time intake was limited to $1200 \mathrm{ml}$ (or more) of water per day. Blood samples were obtained from an antecubital vein between 8 and 9 a.m. on each day of the fast with the subjects in the recumbent position. Simultaneous samples of maternal venous blood and amniotic fluid were drawn from 12 of the pregnant subjects after completion of 84-90 hr of starvation, immediately before intra-amniotic installation of saline. Blood samples were drawn from four additional nonpregnant subjects after an overnight (12 hr) fast only.

L-Alanine was administered in a dose of $0.15 \mathrm{~g} / \mathrm{kg}$ to five of the pregnant and five of the nonpregnant subjects on the morning of the 5th hospital day, after $84 \mathrm{hr}$ of fasting. The alanine was infused intravenously over $3 \mathrm{~min}$ as a $10 \%$ sterile, pyrogen-free solution. Blood samples were obtained from an indwelling needle in an antecubital vein at intervals of $10-30 \mathrm{~min}$ for $30 \mathrm{~min}$ before, and for 180 min after completion of the alanine infusion.

The subjects in the "fed" pregnant group (Table I) were maintained on a 2200-2400 kcal diet. Simultaneous samples of maternal venous blood and amniotic fluid were collected 3-6 hr after ingestion of the breakfast meal immediately before intra-amniotic installation of hypertonic saline.

In both the fed and fasted groups the amniotic fluid was obtained percutaneously (via a spinal needle inserted through the abdominal wall under aseptic conditions), and was free of gross contamination by blood. To minimize further the possibility of contamination by maternal blood, the first $50 \mathrm{ml}$ of amniotic fluid obtained was discarded; analyses were performed on the second $50 \mathrm{ml}$ withdrawn.

Chemical analyses. For analysis of amino acids, heparinized plasma and amniotic fluid were deproteinized with sulfosalicyclic acid (16) immediately after collection. The supernates were stored at $-20^{\circ} \mathrm{C}$ until analysis. Individual free amino acids were determined in the deproteinized supernates by the automated ion-exchange chromatographic technique (17). Glucose was measured in plasma by the glucose oxidase procedure (18).

Statistical analyses were performed according to the method of Snedecor (19). Data on blood glucose, insulin, and ketone acid levels and on urinary nitrogen excretion during the course of the fast in 12 of the pregnant and 6 of the nonpregnant subjects have been reported previously (1).

\section{RESULTS}

Nonpregnant subjects. In Table II the amino acid response to starvation is shown for the nonpregnant subjects. In accordance with previous studies in obese subjects (9), a 2- to 3-fold increment was observed in the branched-chain amino acids, valine, leucine, and iso- 
TABLE II

Plasma Amino Acid Levels during an $84 \mathrm{hr}$ Fast in Nonpregnant Women*

\begin{tabular}{|c|c|c|c|c|}
\hline & \multicolumn{3}{|c|}{ Duration of fast (hr) } & \multirow[b]{2}{*}{$P \ddagger$} \\
\hline & $12(n=11)$ & $60(n=7)$ & $84(n=7)$ & \\
\hline Taurine & $51.5 \pm 2.5$ & $57.9 \pm 3.4$ & $48.9 \pm 6.6$ & NS \\
\hline Threonine & $138.0 \pm 11.3$ & $98.7 \pm 2.5$ & $111.4 \pm 10.0$ & NS \\
\hline Serine & $126.3 \pm 6.0$ & $110.3 \pm 7.2$ & $121.7 \pm 8.2$ & NS \\
\hline Proline & $151.3 \pm 10.1$ & $141.3 \pm 10.0$ & $152.6 \pm 9.7$ & NS \\
\hline Citrulline & $27.2 \pm 1.2$ & $26.4 \pm 2.0$ & $24.0 \pm 2.0$ & NS \\
\hline Glycine & $197.4 \pm 14.6$ & $152.3 \pm 17.9$ & $168.7 \pm 15.0$ & $<0.05$ \\
\hline Alanine & $279.3 \pm 15.1$ & $266.6 \pm 20.1$ & $218.6 \pm 9.4$ & $<0.02$ \\
\hline$\alpha$-Aminobutyrate & $22.0 \pm 3.1$ & $66.0 \pm 3.5$ & $70.6 \pm 4.7$ & $<0.001$ \\
\hline Valine & $201.4 \pm 11.7$ & $410.4 \pm 34.0$ & $407.6 \pm 36.4$ & $<0.005$ \\
\hline Cystine & $94.8 \pm 5.4$ & $109.0 \pm 5.0$ & $99.8 \pm 5.6$ & NS \\
\hline Methionine & $18.0 \pm 1.7$ & $21.7 \pm 1.4$ & $21.4 \pm 1.3$ & $<0.005$ \\
\hline Isoleucine & $51.4 \pm 2.7$ & $151.6 \pm 11.4$ & $155.7 \pm 9.8$ & $<0.001$ \\
\hline Leucine & $99.5 \pm 2.0$ & $266.1 \pm 23.9$ & $260.6 \pm 20.9$ & $<0.001$ \\
\hline Tyrosine & $40.4 \pm 3.3$ & $53.4 \pm 5.6$ & $51.3 \pm 4.4$ & $<0.01$ \\
\hline Phenylalanine & $45.8 \pm 2.4$ & $54.9 \pm 2.5$ & $52.7 \pm 2.0$ & $<0.02$ \\
\hline Ornithine & $71.8 \pm 7.6$ & - & $53.8 \pm 4.6$ & $<0.025$ \\
\hline Lysine & $164.1 \pm 12.1$ & - & $141.8 \pm 13.4$ & $<0.025$ \\
\hline Histidine & $81.4 \pm 5.5$ & - & $74.7 \pm 4.8$ & NS \\
\hline Arginine & $55.5 \pm 6.6$ & - & $43.8 \pm 3.0$ & NS \\
\hline
\end{tabular}

* Data are presented as mean \pm SE in $\mu$ moles/liter. The basic amino acids were not measured in the 60 -hr samples.

$\ddagger P=$ significance of difference between values observed after 12 and $84 \mathrm{hr}$ of fasting (paired $t$ test, $\mathrm{n}=7$ ).

leucine and in $\alpha$-aminobutyrate. Smaller increments were noted in phenylalanine, tyrosine, and methionine. In contrast, a significant decline was observed in the concentrations of the key endogenous glycogenic amino acids $(9,20)$ alanine and glycine. The magnitude of this decline was greatest for alanine. Small decrements in lysine and ornithine were also observed.

Pregnant subjects. The amino acid levels in the pregnant subjects after an overnight $(12 \mathrm{hr}), 60 \mathrm{hr}$, and 84 hr fast are shown in Table III. In the postabsorptive state $(12 \mathrm{hr}$ fast), the levels of 10 of 19 amino acids were significantly reduced below those observed in the nonpregnant group. The mean values of most of the remaining amino acids were also lower in the pregnant group though the changes were not statistically significant. Only in the case of histidine was the mean postabsorptive concentration significantly higher in the pregnant subjects.

In Fig. 1, plasma alanine levels are shown for the pregnant and nonpregnant subjects throughout the fast. Plasma alanine was significantly lower in the pregnant group in the postabsorptive state (12 hr fast) and for the first $60 \mathrm{hr}$ of starvation. Furthermore, whereas a small but significant decline $(P<0.05)$ in plasma alanine occurred in the pregnant group between 12 and 60 $\mathrm{hr}$ of fasting, alanine levels failed to decline significantly in the nonpregnant group until $84 \mathrm{hr}$ of starvation.

In Fig. 2 the changes in plasma glycine induced by starvation in pregnancy are compared with those ob-
TABLE III

Plasma Amino Acid Levels during an $84 \mathrm{hr}$ Fast in Pregnant Women*

\begin{tabular}{|c|c|c|c|c|}
\hline & \multicolumn{3}{|c|}{ Duration of fast (hr) } & \multirow[b]{2}{*}{$P \ddagger$} \\
\hline & $12(n=12)$ & $60(n=12)$ & $84(n=12)$ & \\
\hline Taurine & $36.0 \pm 2.98$ & $43.7 \pm 3.6$ & $42.3 \pm 3.8$ & NS \\
\hline Threonine & $150.9 \pm 7.0$ & $142.3 \pm 9.3$ & $173.5 \pm 12.6$ & $<0.05$ \\
\hline Serine & $107.4 \pm 4.6$ & $128.2 \pm 5.6$ & $132.7 \pm 6.1$ & $<0.001$ \\
\hline Proline & $93.8 \pm 4.9 \S$ & $104.4 \pm 4.1$ & $124.8 \pm 6.2$ & $<0.001$ \\
\hline Citrulline & $18.9 \pm 0.8 \S$ & $17.0 \pm 0.6$ & $17.2 \pm 0.9$ & NS \\
\hline Glycine & $120.8 \pm 7.6 \S$ & $127.3 \pm 9.3$ & $151.3 \pm 13.0$ & $<0.005$ \\
\hline Alanine & $221.6 \pm 9.0$ ป & $200.3 \pm 8.2$ & $203.8 \pm 7.1$ & $<0.05$ \\
\hline$\alpha$-Aminobutyrate & $20.4 \pm 1.6$ & $52.2 \pm 2.3$ & $60.1 \pm 2.6$ & $<0.001$ \\
\hline Valine & $169.8 \pm 3.9 \|$ & $337.2 \pm 12.0$ & $333.5 \pm 17.0$ & $<0.001$ \\
\hline Cystine & $69.1 \pm 3.08$ & $75.0 \pm 0.6$ & $65.3 \pm 2.6$ & NS \\
\hline Methionine & $16.7 \pm 0.7$ & $20.2 \pm 6.1$ & $22.8 \pm 1.6$ & $<0.001$ \\
\hline Isoleucine & $47.0 \pm 1.8$ & $112.8 \pm 5.5$ & $109.7 \pm 6.9$ & $<0.001$ \\
\hline Leucine & $91.0 \pm 2.5 \|$ & $198.8 \pm 8.7$ & $195.0 \pm 12.1$ & $<0.001$ \\
\hline Tyrosine & $34.5 \pm 1.7$ & $42.2 \pm 1.7$ & $44.1 \pm 2.9$ & $<0.005$ \\
\hline Phenylalanine & $42.3 \pm 1.1$ & $49.3 \pm 1.4$ & $49.7 \pm 2.4$ & $<0.005$ \\
\hline Ornithine & $33.7 \pm 5.08$ & - & $33.0 \pm 2.7$ & NS \\
\hline Lysine & $175.8 \pm 13.9$ & - & $153.0 \pm 13.9$ & NS \\
\hline Histidine & $100.7 \pm 5.7 \|$ & - & $79.7 \pm 5.3$ & $<0.005$ \\
\hline Arginine & $51.0 \pm 7.6$ & - & $53.8 \pm 7.9$ & NS \\
\hline
\end{tabular}

* Data are presented as mean $\pm \mathrm{SE}$ in $\mu$ moles/liter. The basic amino acids were not measured in the 60 -hr samples.

$\ddagger P=$ significance of difference between values observed after 12 and $84 \mathrm{hr}$ of fasting (paired $t$ test).

\$ Significantly different from $12 \mathrm{hr}$ value in nonpregnant group $(P<0.001$ unpaired $t$ test).

$\|$ Significantly different from $12 \mathrm{hr}$ value in nonpregnant group $(P<0.025$, unpaired $t$ test).

I Significantly different from $12 \mathrm{hr}$ value in nonpregnant group $(P<0.005$ unpaired $t$ test).

served in nonpregnant subjects and with the changes reported previously in nonpregnant obese subjects fasted for 10 days (9). In the nonpregnant group plasma glycine showed an initial decline and failed to rise above postabsorptive levels during the course of $84 \mathrm{hr}$ of fasting. Similarly, in the obese nonpregnant group, an increment in plasma glycine above basal levels was not observed until after 10 days of starvation. In marked contrast,

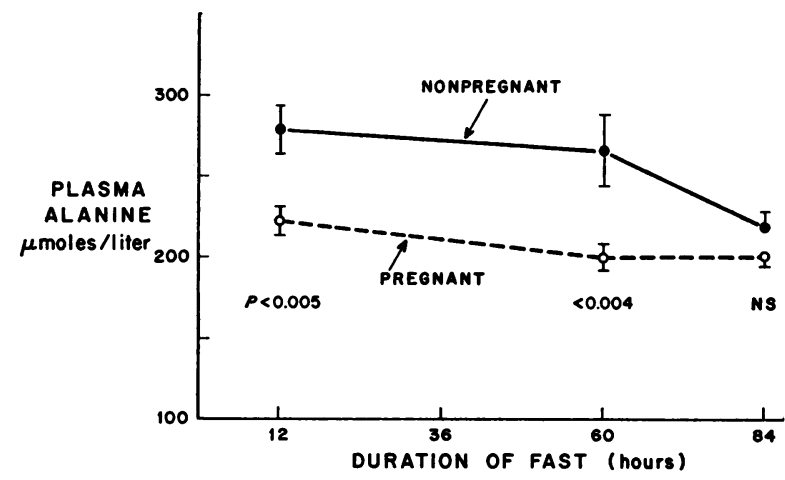

Figure 1 Plasma alanine levels in pregnant and nonpregnant subjects during the course of an $84 \mathrm{hr}$ fast. (Mean $\pm \mathrm{SE}) . P$ values refer to significance of differences between pregnant and nonpregnant subjects. 


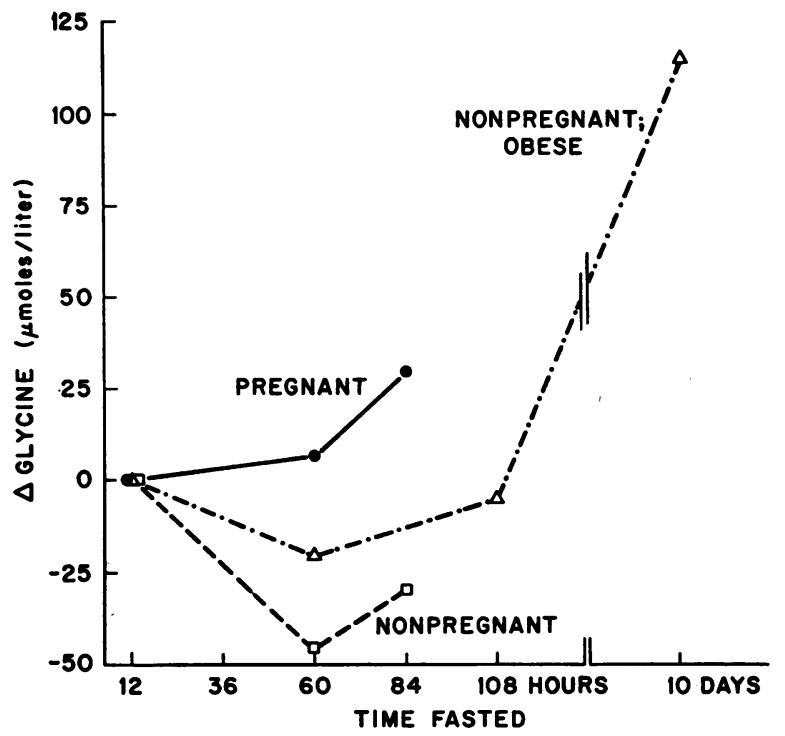

FIGURE 2 Changes in plasma glycine concentration during the course of an $84 \mathrm{hr}$ fast in pregnant and nonpregnant subjects and in previously reported (9), prolonged fasted nonpregnant obese subjects. The mean changes from the basal, postabsorptive state ( $12 \mathrm{hr}$ fast) are shown.

plasma glycine concentration began to rise within $60 \mathrm{hr}$ of fasting in the pregnant group and was significantly elevated by $84 \mathrm{hr}$. A similar acceleration was observed in the pregnant group with respect to starvation-induced increases in plasma threonine and serine. Whereas the concentration of these amino acids failed to rise above basal levels during $84 \mathrm{hr}$ of fasting in the nonpregnant group (Table II), and required 5-10 days of fasting to become elevated in the prolonged fasted obese group (9), a significant elevation was observed after 60-84 hr of caloric deprivation in pregnancy (Table III).

The response of the branched-chain amino acids and $\alpha$-aminobutyrate was generally similar to the nonpregnant group. However, the absolute rise in leucine and isoleucine was smaller in the pregnant group (Table III). Elevations were also observed in methionine, tyrosine, and phenylalanine.

The influence of starvation on amino acid levels in amniotic fluid is shown in Table IV. The concentration of the branched-chain amino acids and $\alpha$-aminobutyrate increased markedly, while alanine levels fell by $25 \%$. Small increments in taurine and serine were also demonstrated. Despite the changes in amino acid levels in amniotic fluid, the relation between amniotic fluid and maternal plasma amino acid concentrations was generally not altered by starvation. Thus the ratios of amniotic fluid to maternal plasma concentrations did not differ in the fed and fasted groups for most amino acids (Table IV). Small decreases were noted however, after fasting in the case of glycine and methionine.
Response to alanine. The effect of alanine administration on plasma glucose levels during prolonged starvation is shown in Fig. 3. Before alanine infusion, plasma glucose concentration after $84 \mathrm{hr}$ of starvation ( -10 and 0 values, Fig. 3 ) was, as anticipated (1), significantly lower in the pregnant group $(P<0.05)$. However, after alanine administration, a comparable increase in plasma glucose was observed in the pregnant and nonpregnant subjects (Fig. 3). In both groups plasma glucose levels rose within the first $10 \mathrm{~min}$, reaching a peak at $50 \mathrm{~min}$. The mean ( $\pm \mathrm{SE}$ ) maximal increase in plasma glucose was $21.2 \pm 2.0 \mathrm{mg} / 100 \mathrm{ml}$ in the nonpregnant group and $24.6 \pm 1.0 \mathrm{mg} / 100 \mathrm{ml}$ in the pregnant subjects.

\section{DISCUSSION}

Previous studies on the amino acid response to starvation in the nonpregnant state have dealt exclusively (9), or primarily (21) with obese individuals. The current data in the nonpregnant group thus provide evidence that the increase in branched-chain amino acids and fall in glycogenic amino acids induced by starvation is not a consequence of the obese state. Furthermore, since the specific amino acids which increased in both the nonpregnant and pregnant groups (valine, leucine, isoleucine, $\alpha$-aminobutyrate, phenylalanine, and tyrosine) correspond to those amino acids which are uniquely sensitive to changes in endogenous insulin levels (22)

TABLE IV

Influence of Fasting on Amino Acid Levels in Amniotic Fluid and on the Ratio of Amniotic Fluid to Maternal Plasma (AF:MP) Amino Acid Concentrations*

\begin{tabular}{|c|c|c|c|c|c|}
\hline & \multicolumn{3}{|c|}{ Amniotic fluid } & \multicolumn{2}{|c|}{ AF:MP } \\
\hline & Fed $\ddagger$ & Fasted $\S$ & $P \|$ & Fed $\ddagger$ & Fasted\& \\
\hline Taurine & $77.2 \pm 5.4$ ฯ & $98.9 \pm 7.8$ & $<0.05$ & 2.4 & 2.3 \\
\hline Threonine & $197.7 \pm 28.2$ & $173.7 \pm 14.2$ & NS & 1.2 & 1.0 \\
\hline Serine & $33.5 \pm 3.4$ & $42.9 \pm 2.6$ & $<0.05$ & 0.3 & 0.3 \\
\hline Proline & $175.2 \pm 12.9$ & $159.9 \pm 9.7$ & NS & 1.5 & 1.3 \\
\hline Citrulline & $12.0 \pm 0.5$ & $8.1 \pm 1.5$ & $<0.02$ & 0.6 & 0.5 \\
\hline Glycine & $169.8 \pm 18.2$ & $151.9 \pm 11.6$ & NS & 1.4 & 1.0 \\
\hline Alanine & $353.0 \pm 21.5$ & $264.8 \pm 17.1$ & $<0.005$ & 1.4 & 1.3 \\
\hline$\alpha-\mathrm{NH}_{2}$ butyrate & $13.4 \pm 2.7$ & $52.7 \pm 2.3$ & $<0.001$ & 0.9 & 0.9 \\
\hline Valine & $136.2 \pm 9.4$ & $255.3 \pm 18.2$ & $<0.001$ & 0.8 & 0.8 \\
\hline Cystine & $77.8 \pm 8.0$ & $72.8 \pm 4.1$ & NS & 1.1 & 1.1 \\
\hline Methionine & $19.2 \pm 2.0$ & $20.3 \pm 1.4$ & NS & 1.2 & 0.9 \\
\hline Isoleucine & $23.8 \pm 3.1$ & $68.3 \pm 5.3$ & $<0.001$ & 0.5 & 0.6 \\
\hline Leucine & $51.7 \pm 5.1$ & $133.4 \pm 11.0$ & $<0.001$ & 0.6 & 0.7 \\
\hline Tyrosine & $43.7 \pm 1.8$ & $46.6 \pm 3.6$ & NS & 1.2 & 1.1 \\
\hline Phenylalanine & $48.5 \pm 1.9$ & $52.2 \pm 3.4$ & NS & 1.2 & 1.1 \\
\hline
\end{tabular}

* The basic amino acids were not measured in the amniotic fluid samples. ¥ Amniotic fluid and maternal plasma were obtained 3-6 hr after the previous meal in subjects maintained on a $2200-2400 \mathrm{kcal}$ diet $(n=6)$.

\& Amniotic fluid and maternal plasma were obtained after 84-90 hr of fasting $(n=12)$.

$\| P=$ significance of differences between mean values in fed and fasted groups (unpaired $t$ test).

I Mean \pm sE, umalęs/liter. 


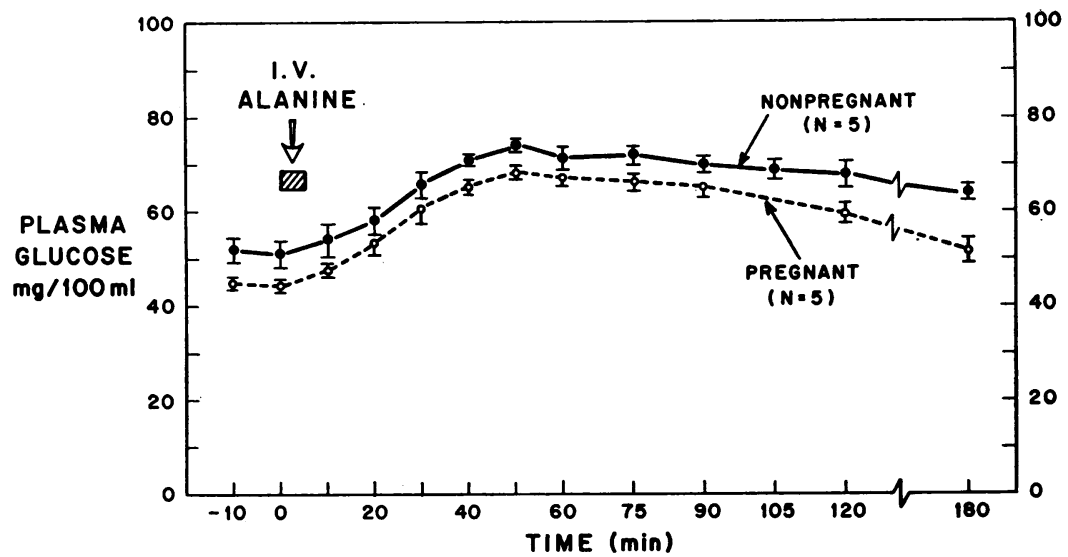

Figure 3 Plasma glucose response to intravenous administration of alanine $(0.15 \mathrm{~g} / \mathrm{kg})$ after $84 \mathrm{hr}$ of starvation in pregnant and nonpregnant subjects. Before alanine administration $(-10$ and $0 \mathrm{~min})$, the glucose levels were significantly lower $(P<0.05)$ in the pregnant group.

and to administration of exogenous insulin (23), the current findings support the conclusion advanced elsewhere (9), that the hyperaminoacidemia of starvation is a consequence of the hypoinsulinemia.

The findings in the pregnant group are of interest with respect to the basal postabsorptive state as well as with regard to the observations during the course of the fast. Although it has long been recognized that pregnancy is characterized by hypoaminoacidemia (24), most of the data on plasma levels of individual amino acids have been obtained on subjects studied in the third trimester (11), often at the time of delivery (25). Little information is available regarding amino acid concentrations at earlier stages of pregnancy (10). Young and Prenton (12) measured maternal plasma amino acid levels in six patients during wk 16-19 of gestation immediately before delivery of the fetus by hysterotomy. Since their patients were anesthetized and in the midst of a surgical procedure the conditions of their study were considerably different from those of the present investigation. The current data indicate that in physically healthy, unanesthetized women, a significant reduction in the postabsorptive levels of most amino acids occurs by wk 16-20 of gestation. In accordance with these findings in humans are recent observations in subhuman primates, in whom gestational hypoaminoacidemia is manifest by midpregnancy with no further reduction in amino acid levels occurring as pregnancy progresses to term (26). The unique increase in postabsorptive levels of histidine in the pregnant group is in keeping with previous demonstrations of relative or absolute hyperhistidinemia during late pregnancy in women (25), and in monkeys studied at various stages of gestation (26).

The effect of pregnancy on the concentration of plasma alanine during starvation is of particular importance since this amino acid has been identified as the primary endogenous glycogenic substrate released by muscle $(27,28)$ and extracted by the liver $(9,20,29)$. In addition, a reduction in plasma alanine has been demonstrated to be of primary importance in the limitation of hepatic gluconeogenesis during prolonged starvation in nonpregnant subjects (9). The current observation that pregnancy accelerates and exaggerates the hypoalaninemic response to starvation suggests that substrate lack contributes to the fasting hypoglycemia characteristic of pregnancy. Lack of endogenous circulating alanine thus may be responsible for the failure of hepatic gluconeogenesis to increase adequately to maintain euglycemia in the face of combined maternal and fetal requirements for glucose. It is noteworthy in this respect that the effect of pregnancy in lowering plasma alanine levels occurs during the first $60 \mathrm{hr}$ of starvation and is coincident with the period in which the exaggerated decline in plasma glucose is most pronounced (1). A similar deficiency in endogenous glycogenic substrate has recently been reported in the fasted pregnant rat (30). In addition, hypoalaninemia comparable to that observed in the pregnant group has been demonstrated to be of primary pathogenetic significance in the syndrome of ketotic hypoglycemia of infancy (31).

Further evidence that lack of glucose precursors rather than altered intrahepatic events is responsible for gestational hypoglycemia is provided by the plasma glucose response to administration of exogenous alanine. When plasma alanine levels were increased in the pregnant group by intravenous infusion of this amino acid, a prompt hyperglycemic response was observed (Fig. 3). Furthermore, despite the lower initial fasting plasma glucose concentrations in the pregnant group, the rapidity with which plasma glucose levels increased and the peak 
increments achieved were equivalent to those observed in the nonpregnant subjects. These findings support the conclusion that maternal hepatic gluconeogenic mechanisms are capable of responding to increased substrate delivery during starvation in pregnancy.

Since alanine has been demonstrated to stimulate glucagon release (32), the question may be raised as to whether glycogenolysis rather than increased gluconeogenesis is responsible for the glycemic response to alanine. That such is not the case in subjects fasted 3-4 days is suggested by the data of Hultman and Nilsson who demonstrated virtually complete depletion of hepatic glycogen reserves during the first $24-48 \mathrm{hr}$ of starvation (33). In addition, studies with isotopically labeled alanine in the overnight and prolonged fasted state have demonstrated that alanine $-{ }^{14} \mathrm{C}$ is promptly incorporated into blood glucose (34).

As to the mechanism of the hypoalaninemia in pregnancy, aminoaciduria has been well documented in gestation (11). However, in contrast to many other amino acids, renal clearance of alanine does not increase in early pregnancy (10). Nevertheless, altered renal handling of alanine during starvation in early pregnancy cannot be excluded on the basis of the current data. Diminished alanine output from maternal protein stores or increased hepatic extraction of alanine could also result in a diminution in circulating levels of this amino acid. However, the former would be expected to result in a reduction and the latter in an augmentation in hepatic gluconeogenesis. The failure to observe any significant difference between pregnant and nonpregnant subjects in the rate of urea nitrogen excretion during starvation (1), supports neither hypothesis. A further possibility is that continuous uptake of alanine by the placenta in the face of preferential utilization of this amino acid for maternal hepatic gluconeogenesis results in depletion of the circulating levels of this substrate. Supporting such a mechanism is the demonstration that active transport of amino acids by the placenta is at least as pronounced in early gestation as in the later stages of pregnancy (25). Accordingly, maternal hypoglycemia, initiated by augmented glucose utilization consequent to fetal dependence on glucose as its primary metabolic fuel (1-3), may be perpetuated by the conceptus' siphoning of glycogenic precursors as well.

The influence of pregnancy on the changes in plasma glycine, threonine, and serine during fasting are of interest inasmuch as these amino acids are unique in demonstrating a delayed increase during prolonged starvation of obese nonpregnant subjects (9). The accelerative effect of pregnancy on the amino acid response to starvation is indicated by the elevations in these amino acids within $60-84 \mathrm{hr}$ of fasting in the pregnant group. As to the mechanism of this increment, hyperglycinemia has been reported in protein-calorie malnutrition (35), and in the mother and neonate in association with restricted maternal protein intake (36). This association of hyperglycinemia with protein lack raises the possibility that the accelerative action of pregnancy on the plasma glycine response to fasting may be a consequence of more rapid depletion of maternal protein reserves resulting from continuous placental transport of amino acids to meet fetal requirements for protein synthesis.

The concentrations of amino acids in amniotic fluid were investigated inasmuch as amniocentesis has been suggested as a useful technique in the antenatal diagnosis of inherited abnormalities of amino acid metabolism and other genetic disorders $(13,14)$. While it is generally advantageous to establish such diagnoses at an early stage of pregnancy the limited quantitative data available on amino acid levels in amniotic fluid have generally been obtained at term $(37,38)$. The current findings in the fed group (Table IV) thus may constitute a useful base line for future comparisons in the prenatal detection of amino acid disorders. Of possibly greater interest is the clear-cut effect of starvation on amino acid levels in amniotic fluid as indicated by a marked increase in valine, leucine, and isoleucine and a decrease in alanine. That these changes were a consequence of altered maternal amino acid levels rather than a result of starvation-induced effects of transport processes in the conceptus is suggested by the generally unaltered ratios of amniotic fluid to maternal plasma amino acid concentrations (Table IV). Moreover the changes in amniotic fluid were restricted to those amino acids demonstrating the greatest absolute perturbations in maternal plasma cencentration during the course of the fast. Regardless of the mechanism involved, the current data indicate that the levels of amino acids in amniotic fluid are profoundly influenced by maternal nutrition. Accordingly, consideration must be given to the preexisting nutritional status of the mother in the antenatal diagnostic evaluation of amino acid concentrations in amniotic fluid.

It should be noted that the column chromatographic technique employed in the current study precluded accurate measurements of glutamine and glutamate (39). The importance of glutamine as a renal substrate is well established (40), while more recent studies have demonstrated muscle release and hepatic uptake of glutamine in postabsorptive man (41). An increase in renal ammoniagenesis and presumably in renal gluconeogenesis has been demonstrated during starvation in pregnancy (1). Whether changes in glutamine metabolism contribute to the altered pattern of gluconeogenesis in pregnancy remains to be determined.

\section{ACKNOWLEDGMENTS}

We thank Thomas Trzaski, Donna Murray, and Marion Holmes for their expert technical assistance, and the nurses 
and staff of the Clinical Research Center for their efforts in caring for our patients. We are also grateful to Dr. Nathan Kase for advice and encouragement throughout the course of this study.

This work was supported in part by U. S. Public Health Service Grants AM 13526 and RR 00125, and by a grant from the Research Foundation of the American Diabetes Association.

\section{REFERENCES}

1. Felig, P., and V. Lynch. 1970. Starvation in human pregnancy: hypoglycemia, hypoinsulinemia and hyperketonemia. Science (Washington). 170: 990.

2. Scow, R. O., S. S. Chernick, and M. S. Brinley. 1964. Hypelipemia and ketosis in the pregnant rat. Amer. $J$. Physiol. 206: 796.

3. Herrera, E., R. H. Knopp, and N. Freinkel. 1969. Carbohydrate metabolism in pregnancy. VI. Plasma fuels, insulin, liver composition, gluconeogenesis, and nitrogen metabolism during late gestation in the fed and fasted rat. J. Clin. Invest. 48: 2260.

4. Kim, Y. J., and P. Felig. 1971. Plasma chorionic somatomammotropin levels during starvation in mid-pregnancy. J. Clin. Endocrinol. Metab. 32: 864.

5. Owen, O. E., P. Felig, A. P. Morgan, J. Wahren, and G. F. Cahill, Jr. 1969. Liver and kidney metabolism during prolonged starvation. J. Clin. Invest. 48: 574.

6. Felig, P., E. Marliss, O. E. Owen, and G. F. Cahill, Jr. 1969. Blood glucose and gluconeogenesis in fasting man. Arch. Intern. Med. 123: 293.

7. Williamson, J. R. 1967. Effects of fatty acids, glucagon and anti-innsulin serum on the control of gluconeogenesis and ketogenesis in rat liver. Advan. Enzyme Regul. 5 : 229.

8. Metzger, B. E., F. S. Agnoli, and N. Freinkel. 1971. Effect of sex and pregnancy on formation of urea and ammonia during gluconeogenesis in the perfused rat liver. Horm. Metab. Res. $2: 367$.

9. Felig, P., O. E. Owen, J. Wahren, and G. F. Cahill, Jr. 1969. Amino acid metabolism during prolonged starvation. J. Clin. Invest. $48: 584$.

10. Christensen, P. J., J. W. Date, F. Schonheyder, and K. Volqvartz. 1957. Amino acids in blood plasma and urine during pregnancy. Scand. J. Clin. Lab. Invest. 9: 54.

11. Zinneman, H. H., U. S. Seal, and R. P. Doe. 1967. Urinary amino acids in pregnancy, following progesterone and estrogen-progesterone. J. Clin. Endocrinol. Metab. 27 : 397.

12. Young, M., and M. A. Prenton. 1969. Maternal and fetal plasma amino acid concentration during gestation and in retarded fetal growth. J. Obstet. Gynaecol. Brit. Commonw. 76: 333 .

13. Nadler, H. L., and A. B. Gerbie. 1970. Role of amniocentesis in the intrauterine detection of genetic disorders. N. Engl. J. Med. 282: 596.

14. Milunsky, A., J. W. Littlefield, J. N. Kanfer, E. H. Kolodny, V. E. Shih, and L. E. Atkins. 1970. Prenatal genetic diagnosis. N. Engl. J. Med. 283: 1441.

15. Felig, P., and Y. J. Kim. Maternal and amniotic fluid substrate levels during caloric deprivation in human pregnancy. Metab. (Clin. Exp.) In press.

16. Block, W. D., M. E. Markovs, and B. F. Steele. 1966. Comparison between free amino acid levels in plasma deproteinated with picric acid and with sulfosalicyclic acid. Proc. Soc. Exp. Biol. Med. 122: 1089.
17. Spackman, D. H., W. H. Stein, and S. Moore. 1958. Automatic recording apparatus for use in the chromatography of amino acids. Anal. Chem. 30: 1190.

18. Huggett, A. S. G., and D. A. Nixon. 1957. Use of glucose oxidase, peroxidase, and $o$-dianisidine in determination of blood and urinary glucose. Lancet. 2: 368.

19. Snedecor, G. W. 1965. Statistical Methods. Iowa State University Press, Ames. 5th edition. 35.

20. Felig, P., and J. Wahren. 1971. Influence of endogenous insulin secretion on splanchnic glucose and amino acid metabolism. J. Clin. Invest. 50: 1702.

21. Adibi, S. A. 1968. Influence of dietary deprivation on plasma concentration of free amino acids in man. $J$. Appl. Physiol. 25 : 52.

22. Felig, P., E. Marliss, and G. F. Cahill, Jr. 1969. Plasma amino acid levels and insulin secretion in obesity. N. Engl. J. Med. 281: 811 .

23. Pozefsky, T., P. Felig, J. Tobin, J. S. Soeldner, and G. F. Cahill, Jr. 1969. Amino acid balance across the tissues of the forearm in postabsorptive man: effects of insulin at two dose levels. J. Clin. Invest. 48: 2273.

24. Plass, E. D., and C. W. Matthew. 1925. Placental transmission. III. The amino acids, non-protein nitrogen, urea and uric acid in fetal and maternal whole blood, plasma and corpuscles. Johns Hopkins Med. J. 36: 393.

25. Ghadimi, H., and P. Pecora. 1964. Free amino acids of cord plasma as compared with maternal plasma during pregnancy. Pediatrics. 33: 500.

26. Kerr, G. R. 1968. The free amino acids of serum during development of Macaca mulatta. II. During pregnancy and fetal life. Pediat. Res. 2 : 493.

27. London, D. R., T. H. Foley, and C. G. Webb, 1965. Evidence for the release of individual amino acids from the resting human forearm. Nature (London). 208: 588.

28. Felig, P., T. Pozefsky, E. Marliss, and G. F. Cahill, Jr. 1970. Alanine: key role in gluconeogenesis. Science (Washington). $167: 1003$.

29. Carlsten, A., B. Hallgren, R. Jagenburg, A. Svanborg, and L. Werkö. 1967. Arterio-hepatic venous differences of fee fatty acids and amino acids. Acta Med. Scand. 181 : 199 .

30. Hare, J. W., B. E. Metzger, and N. Freinkel. 1971. Hypoglycemia during fasting in pregnancy: a substrate deficiency syndrome. Diabetes. 20: 338. (Abstr.)

31. Pagliara, A., I. Karl, D. Devivo, R. Feigin, and D. Kipnis. 1971. Hypoalaninemia : the cause of ketotic hypoglycemia of childhood. J. Clin. Invest. 50: 73a. (Abstr.)

32. Müller, W. A., G. Faloona, and R. H. Unger. 1971. Effect of alanine on glucagon secretion. J. Clin. Invest. $50: 2215$.

33. Hultman, E., and L. H. Nilsson. 1971. Liver glycogen in man. Effect of different diets and muscular exercise. In Muscle Metabolism During Exercise. B. Pernow and B. Saltin, editors. Plenum Publishing Corporation, New York. 143.

34. Felig, P., E. Marliss, T. Pozefsky, and G. F. Cahill, Jr. 1970. Amino acid metabolism in the regulation of gluconeogenesis in man. Amer. J. Clin. Nutr. 23: 986.

35. Saunders, S. J., A. S. Truswell, G. O. Barbezat, W. Wittman, and J. D. L. Hansen. 1967. Plasma free amino acid pattern in protein-calorie malnutrition. Lancet. 2: 795.

36. Lindblad, B. S., R. J. Rahimtoola, M. Said, Q. Haque, and N. Khan. 1969. The venous plasma free amino acid 
levels of mother and child during delivery. III. In a lower socio-economic group of a refugee area in Karachi, West Pakistan, with special reference to the "small for dates" syndrome. Acta Paediat. Scand. 58: 497.

37. Levy, H. L., and P. P. Montag. 1969. Free amino acids in human amniotic fluid. A quantitative study by ionexchange chromatography. Pediat. Res. 3: 113.

38. Emery, A. E. H., D. Burt, M. M. Nelson, and J. B. Scrimgeour. 1970. Antenatal diagnosis and amino acid. composition of amniotic fluid. Lancet. 1 : 1307.
39. Stein, W. H., and S. Moore. 1954. The free amino acids of human blood plasma. J. Biol. Chem. 211: 915.

40. Owen, E. E., and R. R. Robinson. 1963. Amino acid extraction and ammonia metabolism by the human kidney during the prolonged administration of ammonium chloride. J. Clin. Invest. $42: 263$.

41. Marliss, E. B., T. T. Aoki, T. Pozefsky, A. S. Most, and G. F. Cahill, Jr. 1971. Muscle and splanchnic glutamine and glutamate metabolism in postabsorptive and starved man. J. Clin. Invest. 50: 814. 